\title{
A new path planning method for multi-robot formation in three-dimensional space
}

\author{
Quan Zuo ${ }^{1, a}$, Ming Chu ${ }^{1, b}$, Yukun Ding ${ }^{1, c}$, Long Ma ${ }^{1, d}$, Hanxu Sun ${ }^{1, e}$ \\ ${ }^{1}$ Beijing University of Posts and Telecommunications, China Beijing 100876 \\ a hnlszq@yahoo.com, b chuming_bupt@bupt.edu.cn, c dingyukun@bupt.edu.cn \\ d444647811@qq.com, enxsun@bupt.edu.cn
}

\begin{abstract}
Keywords: Three-dimensional space; Multi-robot; Formation control; Tangent circle method Abstract. Aimed at the formation of multi-robot in 3D space, a new path planning method was proposed based on the market auction method and tangent circle method. This method makes use of the market auction method to allocate tasks so that the robot can obtain the coordinates of each task in the low energy consumption and low cost condition of the system. The traditional tangent circle method was improved, and a tangent circle method was proposed to plan the motion path of each robot, and finally form the formation shape and made the robot have the same attitude. The space tetrahedron formation which is formed by four robots is taken as an example for simulation, the results show that the method can realize the path planning of multi-robot formation and the motion attitude control of the robot in three-dimensional space.
\end{abstract}

\section{Introduction}

The formation control method is a multi-robot system in the process of reaching the target position in a formation and keeping control method can adapt to environmental constraints. Because the task and environment of multi-robot formation is different, have formed a multi-robot formation control method, one of the most commonly used methods are leader follower method, virtual structure method and behavior-based method, the graph theory and reinforcement learning method.

The basic idea of leader follower method was composed of multiple robots in formation system, there might be one or more pilot robot (Leader), other none leader robot was the following robot (Follower), followed by the robot relative to the pilot robot position (relative distance L, relative angle $\varphi)$ as the input control variable, followed the relative position of the robot and the robot pilot of infinite approximation of the target value [1]. The leader follower method control structure was relatively simple, but because there was no position feedback between the robot and the robot to follow the pilot, and the leader of the robot was single point control, which was easy to appear behind the robot and so on, the poor robustness of the system [2].

The basic idea of virtual structure method was to regard the system as a virtual rigid body structure, and the coordinate of each robot in the reference coordinate system was the same, that the relative position of the robot was the same [3]. The proposed formation control method for multi-robot a leader - follow method and virtual structure method combined with AUV (Autonomous Underwater Vehicle, autonomous underwater vehicle) the rolling robot motion in 3D space plane and vertical movement, in order to simplify the discussion, that only at the level of AUV plane motion [4].

The basic idea of behavior-based method was to conduct all aspects of multi-robot formation consists of a plurality of basic behaviors of single robot, you can control the method only needs the basic behavior of various combinations of the basic behavior of multi-robot formations. Basic behavior generally included target tracking, avoid obstacles, avoid collisions, formation and formation maintenance, etc. Based on the behavior of the method by the robot mutual perception and control, the system was relatively easy to achieve distributed control, with a very good robustness. But due to the lack of accurate mathematical model analysis, it could not be very effective to ensure the reliability of the system [5].

The method of graph theory was based on graph theory, think of each robot as a node, and nodes in accordance with certain rules were communicated with each other and control commands between the 
nodes according to the direction to 'transfer', the topological structure of the multi-robot system to form a net[6]. Mentioned in the literature flocking and algebraic graph theory based on the idea of considering the coupling of the robot with six degrees of freedom in the three-dimensional space, the multi-robot formation control system was very difficult to achieve, would be taken in three-dimensional space motion was simplified as the horizontal and vertical planes of the two plane formation to analyze the problems in the logic was very clear, but in the actual movement of the decomposition and synthesis was also facing the big problem[7].

The reinforcement learning method was a relatively new research method. The basic idea was to combine the machine learning theory to evaluate the behavior of robots in the learning phase, if the robot's behavior was consistent with the expected reward setting was positive, then the robot will act in the future more use of the behavior, and this behavior the probability will be more and more low, or even disappear. By effectively setting the rules of reward and punishment, each robot could be set for a long time in accordance with the 'training', the final results could be achieved[8].

The artificial potential field method and multi-body dynamics method combining Kane to realized motion planning of AUV in three-dimensional space formation and collaborative control, but the scheme of the kinematic model of vehicle as a particle, considered only three degrees of freedom of movement of each particle, not very good to solve the problem of formation robot system[9].

By comparing the existing multi-robot formation control methods, can be seen on the multi-robot formation control method research were used to solve the two-dimensional plane formation control problem, or only consider the formation shape without considering the attitude of the robots formation. At present, the formation control method in three-dimensional space is simplified as a two-dimensional plane formation problem to deal with, but also in the three-dimensional space formation control algorithm complexity is too high, it is difficult to achieve accurate formation control of multi-robot system. Therefore, the existing formation control method could not be applied to solve the problem of formation control of multi-robot in three dimensional space. In view of the above problems, a new path planning algorithm for multi-robot formation is proposed, which can solve the problem of formation path planning of multi-robot in three dimensional space.

\section{Mathematical model for formation of multi-robot system}

The establishment of mathematical model is the basis of studying the formation control of multi-robot system, including the spatial motion model and the system state description matrix [10]. The establishment of a suitable mathematical model can greatly simplify the mathematical calculation of multi-robot system in formation process.

The spatial motion model of multi-robot system is established in Fig. 1.

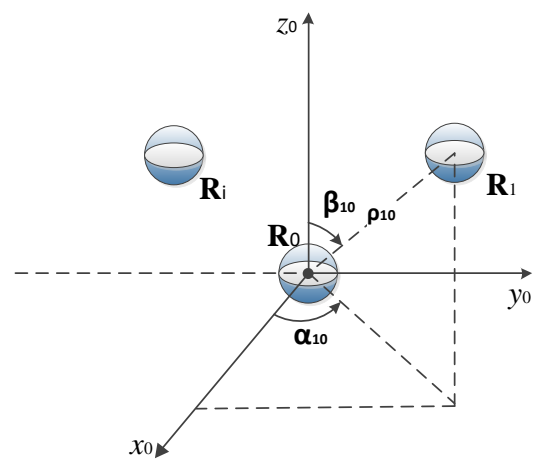

Figure 1. Spatial motion model of multi-robot system

The three-dimensional space position of the robot is obtained by using the reference robot as the origin of the coordinate system, where the position of robot $\mathrm{R}_{\mathrm{i}}$ can represent as $\left[\rho_{i 0}, \alpha_{i 0}, \beta_{i 0}\right]$, current position coordinate matrix of multi-robot system $S_{\mathrm{s}}$ is: 


$$
\boldsymbol{S}_{\mathrm{s}}=\left[\begin{array}{ccc}
0 & 0 & 0 \\
\rho_{10} & \alpha_{10} & \beta_{10} \\
\cdots & \cdots & \cdots \\
\rho_{i 0} & \alpha_{i 0} & \beta_{i 0} \\
\cdots & \cdots & \cdots \\
\rho_{n 0} & \alpha_{n 0} & \beta_{n 0}
\end{array}\right]
$$

$\alpha$ is the angle from the positive $\mathrm{X}$-axis counter clockwise to the projection line, $\alpha \in\left[0,360^{\circ}\right) ; \beta$ is the angle between the $\mathrm{Z}$ clockwise direction axis and the connection of the robots.

The attitude angle of the robot $\mathrm{R}_{\mathrm{i}}$ can through the attitude direction vector $\boldsymbol{r}_{i}$ projection in XOY plane and positive attitude direction vector $r_{i}$ and $\mathrm{Z}$ axis, where $\theta_{i}$ is the angle from $\mathrm{X}$ direction of the axis to the projection of $\boldsymbol{r}_{i}$ in XOY plane, $\varphi_{i}$ is the angle between the $\mathrm{Z}$ axis and the clockwise direction $\boldsymbol{r}_{i}$. Fig. 2 is the model of robot pose angle.

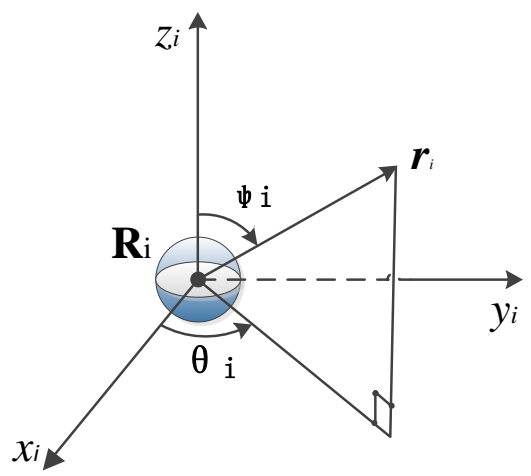

Figure 2. Robot posture angle model diagram

The state matrix $S$ represents the position and attitude of each robot in the multi-robot system, wherein $S$ is defined as follows:

$$
\boldsymbol{S}=\left[\begin{array}{lll}
\boldsymbol{L} & \boldsymbol{q} & \boldsymbol{j}
\end{array}\right]=\left[\begin{array}{ccccc}
0 & 0 & 0 & \theta_{0} & \varphi_{0} \\
\rho_{10} & \alpha_{10} & \beta_{10} & \theta_{1} & \varphi_{1} \\
\cdots & \cdots & \cdots & \cdots & \cdots \\
\rho_{i 0} & \alpha_{i 0} & \beta_{i 0} & \theta_{i} & \varphi_{i} \\
\cdots & \cdots & \cdots & \cdots & \cdots \\
\rho_{n 0} & \alpha_{n 0} & \beta_{n 0} & \theta_{n} & \varphi_{n}
\end{array}\right]
$$

\section{A path planning method for multi-robot formation}

The process of multi-robot formation[11] refers that the state of multiple robots from a disordered state to form a specified formation of initial shape on the premise of human control. The main contents involved in this process are the formation of a task allocation algorithm, path planning algorithm and position control algorithm[12], namely when the system receives formations instructions, how to determine the target position and the method of movement.

The formation process of multi-robot formation is divided into two steps. Firstly, the method of task allocation is studied in the process of forming formation, a market auction method is proposed to optimize the allocation of tasks, and it is applied to the formation task allocation of multi robots, to solve the coordinate assignment problem in the forming formation process. Secondly, the path planning method based on tangent circle method is proposed for multi robot formation process in three dimensions space. 
Logical block diagram of multi-robot forming formation process. The logic diagram of multi-robot forming formation process is shown in Fig. 3.

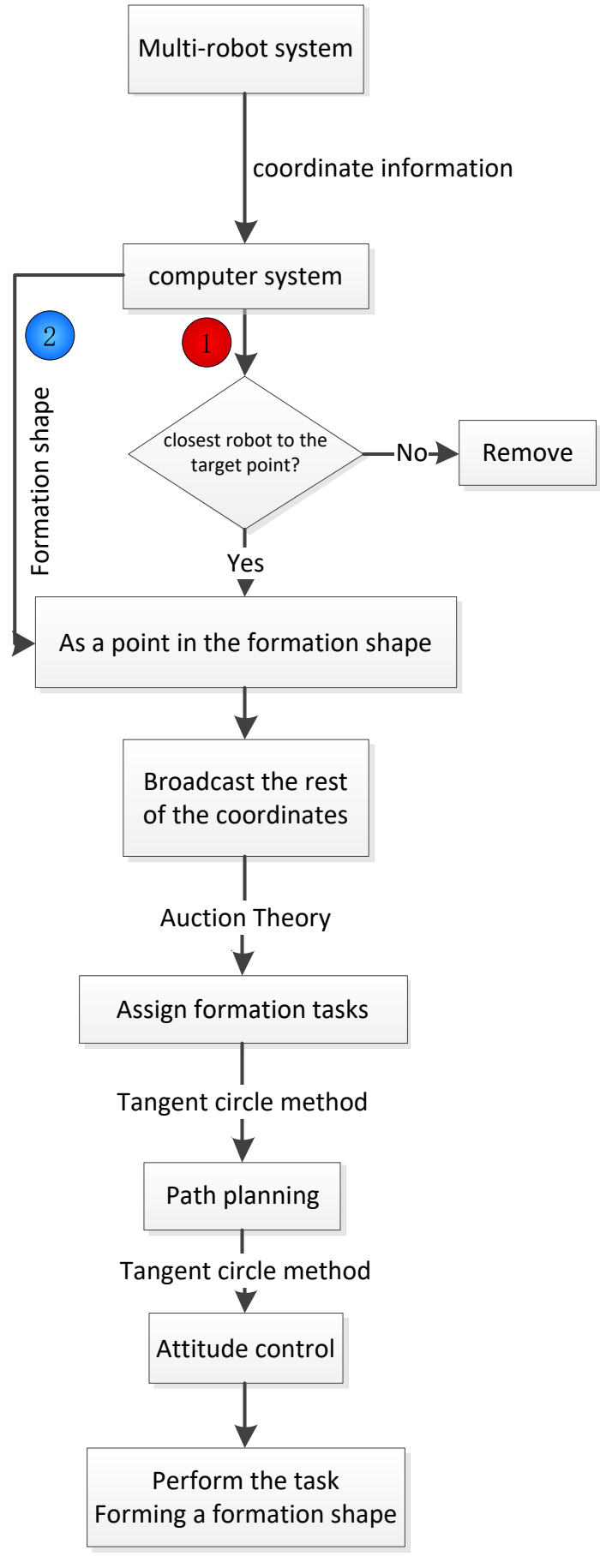

Figure 3. The logical block diagram of multi-robot formation

The task allocation of multi-robot formation process based on market auction method. By means of market auction method, the target state matrix $\boldsymbol{S}_{\mathrm{T}}$ is able to assign the coordinates to each robot, and form a path to track the reference path, each robot can move to the target position in the formation. The traditional method of auction market[13] only according to the target point and the distance between the robots to coordinate the distribution, when the number of robots in the multi robot system is relatively low, the coordinate distribution errors of traditional method is relatively small, but when the number of robots increased significantly, the traditional method is likely to result in the forming formation of multi-robot system needs longer time, more energy consumption. 
This paper proposes a modified market auction algorithm, to calculate the distribution of all coordinates in multi-robot formation system, comprehensively considerate the sum of distance for all robots Sumdistance to the formation of target movement, from receiving instruction to forming formation, the time $T_{\text {cost }}$ required by the robot(the maximum sum of each robot attitude adjustment time $T_{a}$ and robot motion to the specified position time $T_{b}$ ), Sumdistance and $T_{\text {cost }}$ are used to set the weights parameters of $K_{a}$ and $K_{b}$ in different environments can choose different weight parameters.

In order to ensure the stability of formation shape, when forming a formation, each robot needs to have the same speed $V_{g}$ and attitude angle $\theta_{g}, \varphi_{g}$, so it is necessary to calculate the minimum $\min \left\{T_{i}\right\}$ of the time $T_{i}$ required for each robot to move to a specified point in the coordinates. Assuming the robot angular velocity is $\omega, L_{i}$ is the movement distance for the robot $R_{i}$ in the forming formation process, so

$$
T_{i}=\frac{\left|\Delta \theta_{i}\right|+\left|\Delta \varphi_{i}\right|}{\omega}+\frac{L_{i}}{v_{\max }}
$$

As a result $T_{\text {cost }}=\max \left\{T_{i}\right\}$, the average speed of each robot motion to the specified target point:

$$
\overline{v_{i}}=\frac{S_{i}}{T_{\cos t}-\frac{\left|\Delta \theta_{i}\right|+\left|\Delta \varphi_{i}\right|}{\omega}}
$$

Therefore, according to the target speed $V_{g}$ and motion time $T_{\text {cost }}$, combined with the dynamic equations of the robot, carry out the kinematics control to robot speed, make each robot can reach the designated position in the specified time with accurate speed and attitude.

Hypothesis formation efficiency index in the process of formation for $\eta$, so:

$$
\eta_{k}=K_{a} \cdot \frac{n ! \cdot \text { Sumdistance }_{k}}{\sum_{k=1}^{n !} \text { Sumdistance }_{k}}+K_{b} \cdot \frac{n ! \cdot T_{\cos t k}}{\sum_{k=1}^{n !} T_{\cos t k}}
$$

$k$ represents the kth coordinate distribution scheme, $0 \leq \eta_{k} \leq 2$.

By setting the appropriate weight parameters $K_{a}$ and $K_{b}$, we can choose the different formation distribution scheme as the optimal solution.

In the spherical coordinate system, the spherical coordinates of A and B of two robots are known as $\left[\begin{array}{lll}\rho_{a} & \alpha_{a} & \beta_{a}\end{array}\right],\left[\begin{array}{lll}\rho_{b} & \alpha_{b} & \beta_{b}\end{array}\right]$, the rectangular coordinates can be expressed as

$$
\left\{\begin{array}{c}
x_{a}=\rho_{a} \sin \beta_{a} \cos \alpha_{a} \\
y_{a}=\rho_{a} \sin \beta_{a} \sin \alpha_{a} \\
z_{a}=\rho_{a} \cos \beta_{a}
\end{array},\left\{\begin{array}{c}
x_{b}=\rho_{b} \sin \beta_{b} \cos \alpha_{b} \\
y_{b}=\rho_{b} \sin \beta_{b} \sin \alpha_{b} \\
z_{b}=\rho_{b} \cos \beta_{b}
\end{array}\right.\right.
$$

Therefore, we can get the distance between $\mathrm{A}$ and $\mathrm{B}$ points:

$$
|\mathbf{A B}|=\sqrt{\left(x_{a}-x_{b}\right)^{2}+\left(y_{a}-y_{b}\right)^{2}+\left(z_{a}-z_{b}\right)^{2}}
$$

Method for path planning and pose control of multi-robot formation process based on the tangent circle method. Optimally, each robot in the multi-robot system achieves the required pose and forms the formation shape in a short time, and moves toward the target area of the system at a same movement speed, when forming the formation shape. Therefore, the problems faced in the process of forming the formation of the multi-robot system includes path planning and pose control problems when each robot moves to the target coordinates of formation.

The tangent circle method [14] in two-dimensional plane can be applied to robot path planning and pose control in combination with the method for pose control of wheeled robots. The tangent circle method is shown in Fig. 4. 


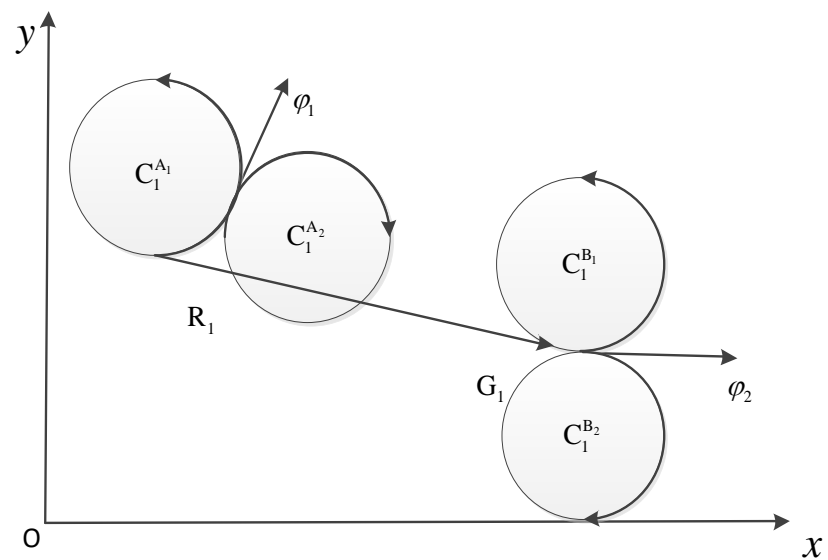

Figure 4. The tangent circle method schematic diagram in two-dimensional plane

Two circles are respectively made at the starting point and the target point with the minimum turning radius, one to the left and the other to the right, and the orientation lines of the starting point and the target point of the robot are the common tangent of the two circles, respectively. Firstly, the robot rotates along circle $C_{1}^{A}$, then moves along the common tangent of circle $C_{1}^{A}$ and circle $C_{1}^{B}$ to circle $\mathrm{C}_{1}^{\mathrm{B}}$, and finally rotates around circle $\mathrm{C}_{1}^{\mathrm{B}}$ to reach the target point at a desired angle. Similarly, the four circles can form four different completion paths. To complete the robot position and path control process, it only needs to calculate the shortest path in an actual situation. Point $\mathrm{G}_{1}$ is the target location for robot $\mathrm{R}_{1}$ to reach.

The path planning method based on the tangent circle method is used to solve the problems of the path planning of two-dimensional plane. The existing tangent circle algorithm needs to be improved so that the algorithm can solve the problems of robot path planning in the three-dimensional space.

The tangent circle method is improved, which can be used to solve the path planning and pose control of multi-robot formation process in the three-dimensional space. The tangent circle method in the three-dimensional space is shown in Fig. 5.

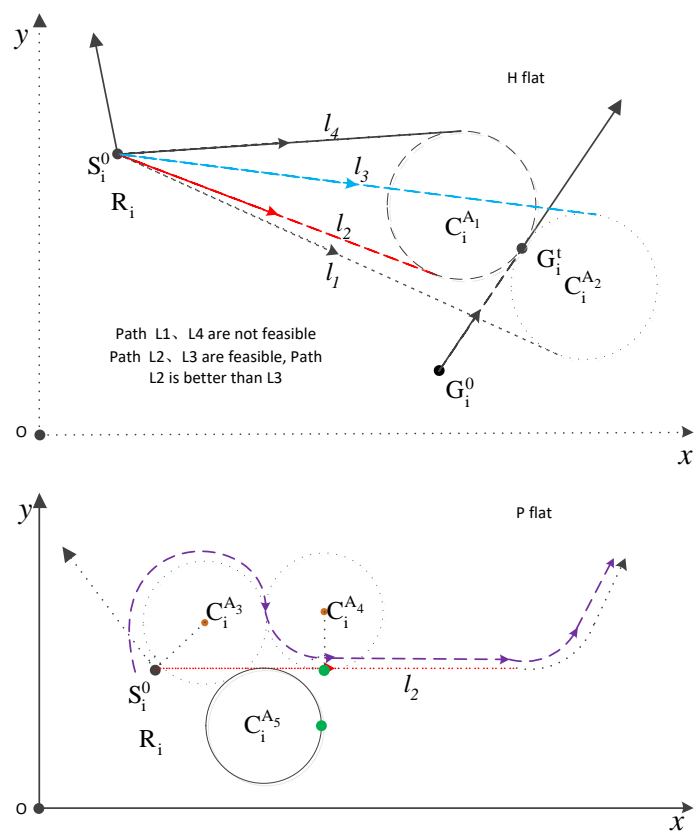

Figure 5. The tangent circle method in the three-dimensional space

Step 1: Calculate the time $t_{i}$ required for the robot $\mathrm{R}_{\mathrm{i}}$ to reach the specified target position by the program algorithm; 
Step 2: By using $G_{i}^{t}$ to represent the target point of the robot $\mathrm{R}_{\mathrm{i}}$, plot the position of $G_{i}^{t}$ in the drawing, then combined with the target location of attitude angle direction vector $\boldsymbol{r}_{\mathrm{i}}$ and robot $\mathrm{R}_{\mathrm{i}}$ 's initial position coordination $\mathrm{S}_{\mathrm{i}}^{0}$ can build a plane $\mathrm{H}$; In the $\mathrm{H}$ plane, using $G_{i}^{t}$ as the cut-off point, constructing two circle $\mathrm{C}_{\mathrm{i}}^{\mathrm{A}_{1}}$ and $\mathrm{C}_{\mathrm{i}}^{\mathrm{A}_{2}}$ about the target point trajectory line $G_{i}^{0} G_{i}^{t}$, preset turning radius $r_{i}$ of robot $\mathrm{R}_{\mathrm{i}}$ is outer radius;

Step 3: In the plane $H$, make a tangent from the initial position $S_{i}^{0}$ of the robot to $C_{i}^{A_{1}}, C_{i}^{A_{2}}$, it is easy to know that there are four solution paths straight $l_{1}, l_{2}, l_{3}, l_{4}$, the straight line $l_{2}, l_{3}$ are satisfied to the robot kinematics, we can easy to know that $l_{2}$ is path optimization by calculation;

Step 4: Through the initial position $\mathrm{S}_{\mathrm{i}}^{0}$ of the robot $\mathrm{R}_{\mathrm{i}}$, initial attitude angle, and linear $l_{2}$ can get a plane $\mathrm{P}$, denoted as the plane $\mathrm{P}$, you need to find a path in the plane $\mathrm{P}$ makes the robot $\mathrm{R}_{\mathrm{i}}$ can go along this path to enter the $l_{2}$;

Step 5: In the $\mathrm{P}$ plane, do circle $\mathrm{C}_{\mathrm{i}}^{\mathrm{A}_{3}}$ in the straight side $l_{2}$ through the $\mathrm{S}_{\mathrm{i}}^{0}, r_{i}$ is the radius of the circle; In linear $l_{2}$ on both sides of the up and down, make a circle $\mathrm{C}_{\mathrm{i}}^{\mathrm{A}_{4}}$, circle $\mathrm{C}_{\mathrm{i}}^{\mathrm{A}_{5}}$, tangent to circle $\mathrm{C}_{\mathrm{i}}^{\mathrm{A}_{3}}$ and line $l_{2}$ respectively, as shown in Fig. 5, only circle $\mathrm{C}_{\mathrm{i}}^{\mathrm{A}_{4}}$ meet the requirements, therefore, the path can be constructed as shown in the dotted line.

Take $S_{1}^{0}:\left[\begin{array}{lllll}0 & 0 & 0 & \theta_{1} & \varphi_{1}\end{array}\right], G_{1}^{0}:\left[\begin{array}{lllll}0 & 0 & 0 & 0 & \frac{\pi}{2}\end{array}\right]$ as an example to calculate the path curve.

Through the $x$-axis, $\mathrm{S}_{\mathrm{i}}^{0}$-point and $\boldsymbol{r}_{\mathrm{i}}$ can be drawn plane $\mathrm{R}_{1}^{\mathrm{P}}$, the space plane equation solution:

The normal vector of plane $\mathrm{R}_{1}^{\mathrm{P}}: \boldsymbol{n}_{\mathbf{R}_{\mathbf{1}}^{\mathrm{p}}}=\boldsymbol{n}_{x} \times \boldsymbol{r}_{\mathbf{i}}=\left[\begin{array}{ccc}\boldsymbol{i} & \boldsymbol{j} & \boldsymbol{k} \\ 1 & 0 & 0 \\ 1 & \tan \theta \frac{1}{\cos \theta \tan \varphi}\end{array}\right]$.

The equation for plane $\mathrm{R}_{1}^{\mathrm{P}}$ is:

$$
-\frac{1}{\cos \theta \tan \varphi} \cdot y+\tan \theta \cdot z=0
$$

Suppose the angle between $\boldsymbol{r}_{\mathrm{i}}$ and $\boldsymbol{n}_{x}$ is $\delta$, then:

$$
\delta=\arccos \frac{\boldsymbol{r}_{\mathbf{i}} \cdot \boldsymbol{n}_{x}}{\left|\boldsymbol{n}_{\mathbf{i}}\right| \cdot\left|\boldsymbol{n}_{x}\right|}=\arccos (\cos \theta \sin \varphi)
$$

In the XOY plane, draw a straight line with the direction of $\delta$, as shown in Fig. 6, and draw the tangent circle $\mathrm{C}_{1}^{\mathrm{A}_{1}}$.

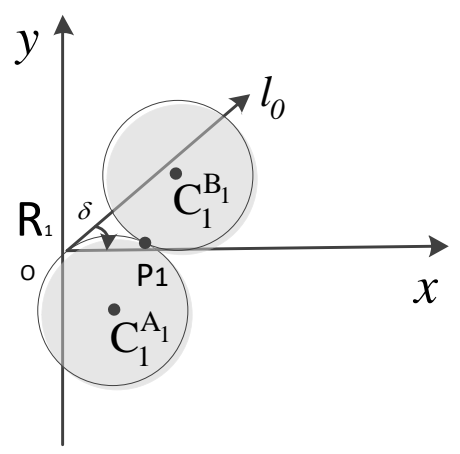

Figure 6. Geometrical relations between $\mathrm{C}_{1}^{\mathrm{A}_{1}}$ and $\mathrm{C}_{1}^{\mathrm{B}_{1}}$ on plane XOY 
The equation for circle $\mathrm{C}_{1}^{\mathrm{A}_{1}}$ can be written as:

$\left\{\begin{array}{c}(x-r \sin \delta)^{2}+(y+r \cos \delta)^{2}=r^{2} \\ z=0\end{array}\right.$

$r$ is the turning radius.

The center $(a, b)$ of the circle $C_{1}^{B_{1}}$ is defined by:

$$
\left\{\begin{array}{c}
(a-r \sin \delta)^{2}+(b+r \cos \delta)^{2}=4 r^{2} \\
a>r \sin \delta \\
b \cos \delta>0 \\
|b|=r
\end{array}\right.
$$

The equation for circle $\mathrm{C}_{1}^{\mathrm{B}_{1}}$ can be written as:

$\left\{\begin{array}{c}(x-a)^{2}+(y-b)^{2}=r^{2} \\ z=0\end{array}\right.$

The coordinates of tangent point $\mathrm{P}_{1}$ are defined as follows:

$\left\{\begin{array}{l}x=\frac{r \sin \delta+a}{2} \\ y=\frac{-r \cos \delta+b}{2} \\ z=0\end{array}\right.$

Suppose the angle between plane $\mathrm{R}_{1}^{\mathrm{P}}$ and plane $\mathrm{XOY}$ is $\gamma$, then:

$$
\gamma=\arccos \frac{\left|\boldsymbol{n}_{\mathrm{R}_{1}^{\mathrm{P}}} \cdot \boldsymbol{n}_{z}\right|}{\left|\boldsymbol{n}_{\mathrm{R}_{1}^{\mathrm{P}}}\right| \cdot\left|\boldsymbol{n}_{z}\right|}
$$

Then by the coordinate rotation formula, after the rotation around the $\mathrm{x}$-axis to meet the new coordinates:

$\left\{\begin{array}{l}x^{\prime}=x \\ y^{\prime}=y \cos \gamma-z \sin \gamma \\ z^{\prime}=y \sin \gamma+z \cos \gamma\end{array}\right.$

The equation for the circle $\mathrm{C}_{1}^{\mathrm{A}_{1}^{\mathrm{P}}}$ in $\mathrm{R}_{1}^{\mathrm{P}}$ plane is:

$$
\left\{\begin{array}{c}
(x-r \sin \delta)^{2}+\left(\frac{y}{\cos \gamma}+r \cos \delta\right)^{2}=r^{2} \\
z=\frac{y}{\sin \gamma}
\end{array}\right.
$$

The equation for circle $\mathrm{C}_{1}^{\mathrm{B}_{1}^{\mathrm{P}}}$ is:

$$
\left\{\begin{array}{c}
(x-a)^{2}+\left(\frac{y}{\cos \gamma}-b\right)^{2}=r^{2} \\
z=\frac{y}{\sin \gamma}
\end{array}\right.
$$




\section{Simulation verification}

Simulation of task assignment algorithm for four robots formation process based on market auction. Definition of the coordinate and attitude of the robot are represented by matrix $\boldsymbol{A}_{i}=\left[\begin{array}{lllll}x_{i} & y_{i} & z_{i} & \theta_{i} & \varphi_{i}\end{array}\right], \mathrm{x} / \mathrm{y} / \mathrm{z}$ represents the position of the robot in cartesian coordinate system, and $\theta, \varphi$ represents the orientation of the robot in the cartesian coordinate system. Take that four robots forming a tetrahedron-shaped multi-robot formation as an example, the initial coordinates are:

$$
\left[\begin{array}{l}
\mathrm{R}_{0} \\
\mathrm{R}_{1} \\
\mathrm{R}_{2} \\
\mathrm{R}_{3}
\end{array}\right]=\left[\begin{array}{ccccc}
4 & 3 & 8 & 32^{\circ} & 2^{\circ} \\
2 & 5 & 6 & 64^{\circ} & 58^{\circ} \\
2 & -4 & 9 & 20^{\circ} & -30^{\circ} \\
-3 & 2 & 6 & 160^{\circ} & 44^{\circ}
\end{array}\right] .
$$

And the coordinates of each vertex of the target formation shape are:

$$
\left[\begin{array}{c}
\mathrm{G}_{0} \\
\mathrm{G}_{1} \\
\mathrm{G}_{2} \\
\mathrm{G}_{3}
\end{array}\right]=\left[\begin{array}{ccccc}
10 & 10 & 10 & 30^{\circ} & 50^{\circ} \\
13 & 10 & 10 & 30^{\circ} & 50^{\circ} \\
10 & 13 & 10 & 30^{\circ} & 50^{\circ} \\
10 & 10 & 13 & 30^{\circ} & 50^{\circ}
\end{array}\right] \text {. }
$$

All the task allocation methods in the four-robot formation system are shown in Table 1.

Tab.1 All the task allocation methods in the four-robot formation system

\begin{tabular}{|c|c|c|c|}
\hline$k$ & Sumdistance $\mathbf{( m )}$ & $T_{\text {cost }} \mathbf{( s )}$ & $\eta$ \\
\hline 1 & 44.9762 & 20.5921 & 0.8845 \\
\hline 2 & 46.0676 & 21.4316 & 0.9133 \\
\hline 3 & 52.5190 & 21.5433 & 0.9783 \\
\hline 4 & 51.6614 & 24.6755 & 1.0382 \\
\hline 5 & 50.6772 & 24.6755 & 1.0286 \\
\hline 6 & 52.6262 & 21.5433 & 0.9793 \\
\hline 7 & 46.0676 & 20.5921 & 0.8950 \\
\hline 8 & 47.1590 & 21.4316 & 0.9239 \\
\hline 9 & 51.6614 & 20.5921 & 0.9493 \\
\hline 10 & 53.1851 & 24.7952 & 1.0556 \\
\hline 11 & 52.2009 & 24.7952 & 1.0460 \\
\hline 12 & 51.7686 & 21.4316 & 0.9686 \\
\hline 13 & 50.6772 & 20.5921 & 0.9397 \\
\hline 14 & 52.2009 & 24.7952 & 1.0460 \\
\hline 15 & 52.6262 & 21.5433 & 0.9793 \\
\hline 16 & 51.7686 & 24.6755 & 1.0392 \\
\hline 17 & 56.3782 & 24.6755 & 1.0839 \\
\hline 18 & 58.7594 & 24.7952 & 1.1096 \\
\hline 19 & 50.2268 & 24.7952 & 1.0269 \\
\hline 20 & 49.7946 & 21.4316 & 0.9495 \\
\hline 21 & 57.7696 & 24.7952 & 1.1000 \\
\hline 22 & 55.3884 & 24.6755 & 1.0743 \\
\hline 23 & 49.7946 & 24.6755 & 1.0201 \\
\hline 24 & 51.7435 & 21.5433 & 0.9708 \\
\hline
\end{tabular}

From the data in the table, we can know that the system has the optimal solution when $\mathrm{I}=0.8845$, which cost $20.5921 \mathrm{~s}$ and the total movement of which is $44.9762 \mathrm{~m}$.

The distribution of the task shown in Fig. 7 can be obtained through MATLAB simulation, in which the solid dots are the initial position of each robot, and The hollow dots are the target points' position. 


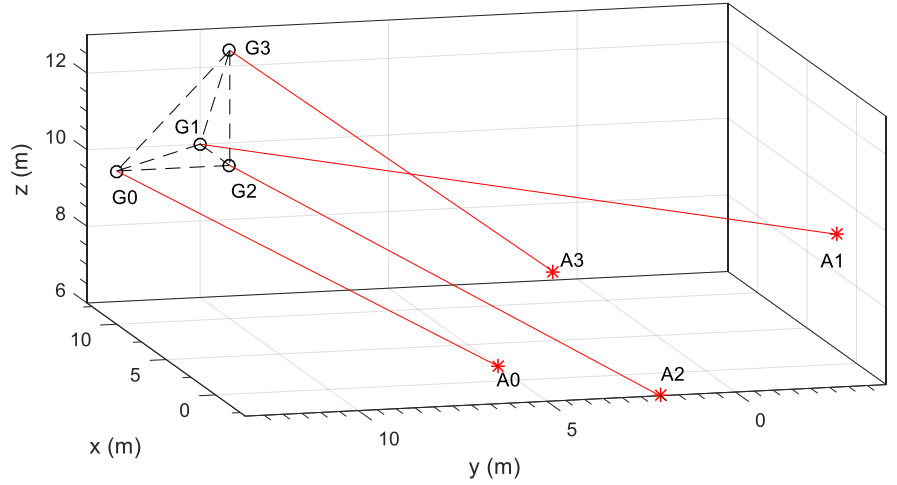

Figure 7. Simulation of formation task assignment

This solution is the optimal solution for a given initial state and target position, which The proved effectiveness of the task allocation algorithm for multi-robot formation process.

Tangent circle method based algorithm simulation for the path planning and pose control of four robots formation process. Take tetrahedron formation as an example, the eventually formed tetrahedron formation shape is shown as the tetrahedron $G_{0} G_{1} G_{2} G_{3}$ in the Fig. 8, and moves in the positive direction of the axis $x$ toward the target point Gt.

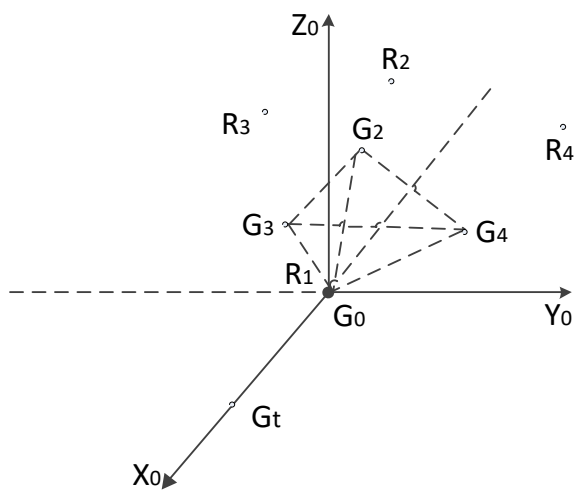

Figure 8. Space tetrahedral formation model

The target status matrix:

$$
\boldsymbol{S}_{\mathrm{T}}=\left[\begin{array}{ccccc}
0 & 0 & 0 & \theta & \varphi \\
5 & 150^{\circ} & 90^{\circ} & \theta & \varphi \\
5 & 180^{\circ} & 35.26^{\circ} & \theta & \varphi \\
5 & 210^{\circ} & 90^{\circ} & \theta & \varphi
\end{array}\right] .
$$

The problem of the formation process transforms to use a algorithm to make $S_{\mathrm{T}}=S$. In order to ensure the unity of the target matrix and ease of calculating, we assume that the row vectors of the target matrix are determined by the magnitude of $\boldsymbol{S}_{\mathrm{i} 1}^{\mathrm{T}}, \boldsymbol{S}_{\mathrm{i} 2}^{\mathrm{T}}, \boldsymbol{S}_{\mathrm{i} 3}^{\mathrm{T}}$, and the row vector with small column coordinates precedes.

Through the coordinate assignment of multi-robot formation based on improved market auction method studied in the section 3.2, we can assign the coordinates of the formation shape $G_{1}, G_{2}, G_{3}$ to robots $\mathrm{R}_{1}, \mathrm{R}_{2}, \mathrm{R}_{3}$. 
Through the tangent circle method based algorithm simulation for the path planning and pose control of four - robot formation process studied in the section 3.3, we can calculate the formation path of each robot, and the final simulation diagram is shown as Fig. 9.

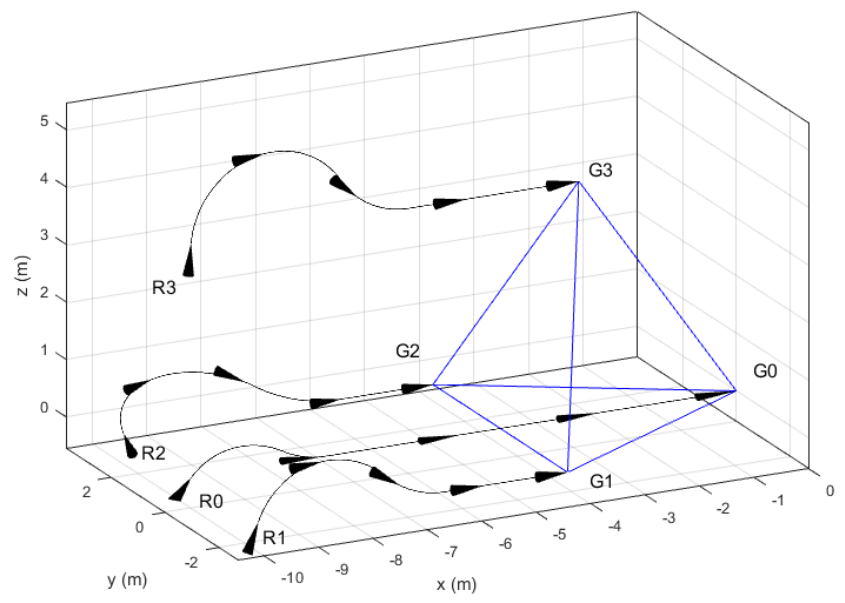

Figure 9. Simulation of tetrahedron formation shape

From Fig. 9, it is shown that in the case where the initial attitude directions of the respective robots are different, the tangent circle method can be used to plan out the movement route and adjust the movement posture, and finally form a tetrahedral formation shape with other robots with the same motion posture, which proved the effectiveness of the path planning and pose control algorithm in the multi-robot formation process.

\section{Conclusions}

To study the task allocation problem in the process of multi-robot system formation, this paper proposed a formation task assignment method based on improve the market auction method, which can obtain the optimal solution in all the task allocation schemes. In the problem of formation path planning in multi-robot system, we improved the tangent circle method used in the traditional two-dimensional plane, which makes the new method able to well solve the problem of path planning and pose control in the process of multi-robot formation in three-dimensional space. Finally, we tested and verified the effectiveness of the algorithm of this paper through simulation.

\section{Acknowledgements}

This work is supported by National Natural Science Foundation of China(Grant No.51305039) and National Basic Research Program of China(973 Program, Grant No. 2013CB733000).

\section{References}

[1]. Shao J, Xie G, Wang L. Leader-following formation control of multiple mobile vehicles[J]. IET Control Theory and Applications, 2007, 1(2): 545-552.

[2]. Xiaoli, Wang, Wei, Ni, Xinsheng, Wang. Leader-Following Formation of Switching Multi-robot Systems via Internal Model[J]. IEEE Transactions on Systems, Man, and Cybernetics, Part B (Cybernetics), 2012, 3(42): 817-826.

[3]. Cammy, K, Peterson, Jeff, Barton. Virtual structure formations of cooperating UAVs using wind-compensation command generation and generalized velocity obstacles[J]. 2015 IEEE Aerospace Conference, 2015, 1(3): 1-7.

[4]. Yuan Jian. Formation control of multi-autonomous underwater vehicles in network environment[D].Qingdao: Ocean University of China, 2011. 
[5]. Gianluca, Antonelli;, Filippo, Arrichiello;, Stefano, Chiaverini. Experiments of Formation Control With Multi-robot Systems Using the Null-Space-Based Behavioral Control[J]. IEEE Transactions on Control Systems Technology, 2009, 17(5): 1173-1182.

[6]. Hua, Wang;, Yi, Guo. Minimal persistence control on dynamic directed graphs for multi-robot formation[J]. Robotics and Automation (ICRA), 2012 IEEE International Conference on, 2012, (1): $1557-1563$.

[7]. Jiang L M . Research on multi-UAV formation control and cooperative mechanism under weak communication[D]. Haerbin: Harbin Engineering University, 2012.

[8]. Hitoshi, Iima;, Yasuaki, Kuroe. Swarm reinforcement learning methods improving certainty of learning for a multi-robot formation problem[J]. 2015 IEEE Congress on Evolutionary Computation (CEC), 2015, (1): 3026-3033.

[9]. Yang Yan. Research on formation motion and stability of underwater vehicle formation[D]. Tianjin: Tianjin University, 2012.

[10]. Miguel, Aranda;, Gonzalo, López-Nicolás;, Carlos, Sagüés;, Michael, M, Zavlanos. Three-dimensional multi-robot formation control for target enclosing[J].2014 IEEE/RSJ International Conference on Intelligent Robots and Systems, 2014, (1): 357-362.

[11]. Fan S B. Research on multi-robot formation control[D]. Haerbin: Harbin Engineering University, 2008.

[12]. Wu X P, Feng Z P, Zhu J M. A new method of AUVs formation control [J]. Ship Science and Technology, 2008, 30(2): 128-134.

[13]. Zhai H C . Research on formation control of Multi-bionic robotic[D]. Qingdao: Qingdao University, 2014.

[14]. Yang X X . Research on point stabilization and formation control of autonomous robot[D]. Qingdao: Ocean University of China, 2012. 